\title{
MODELOS CONCEPTUALES SOBRE LAS RELACIONES ENTRE DIGESTIÓN, RESPIRACIÓN Y CIRCULACIÓN
}

\author{
NÚÑEZ, F. y BANET, E. \\ Facultad de Educación. Departamento de Didáctica de las Ciencias Experimentales. \\ Universidad de Murcia. Campus de Espinardo s/n. 30100 Murcia.
}

\begin{abstract}
SUMMARY
Conceptual patterns of the relations digestion/circulatory system and breathing/circulatory system, found in a sample of non-university students whose ages range between 11 and 17 years old are described in this work. These patterns show that students' conceptions are relatively organized in their conceptual structure and in spite of being sometimes incomplete and wrong, they seem to be coherent for them, even making up real theories that they use to explain any problematic situation connected with these processes.
\end{abstract}

\section{INTRODUCCIÓN}

Aunque a lo largo de las últimas décadas han sido numerosos los trabajos destinados a explorar las concepciones de Ias alumnas y los alumnos sobre diferentes aspectos de las ciencias experimentales, en la mayoría de los casos se ha dado la sensación de que se trata de ideas aisladas e inconexas entre sí, ligadas -por otra parte- a la especificidad del dominio conceptual objeto de analisis. Ésta ha sido una de las razones argumentadas por algunos autores (Pozo, Gómez Crespo, Limón y Sanz Serrano, 1991) para señalar la heterogeneidad de las investigaciones surgidas en torno a lo que se ha denominado «movimiento de las concepciones alternativas» (término acuñado por Gitbert y Swift, 1985).

Sin embargo, aunque constituya una línea aún no muy desarrollada pero necesaria, desde diversas perspectivas se ha puesto de manifiesto que, con respecto a un tópico determinado, buena parte de las concepciones que poseen las y los estudiantes se organizan en torno a estructuras conceptuales más o menos relacionadas entre sí, en la mayoría de los casos, organizadas jerárquicamente. Así se desprende, por ejemplo, de la teoría de Ausubel (1978) sobre la organización del conocimiento, de las afirmaciones de Rodrigo (1985) o Pozo y otros (1991) con respecto a lo que denominan «teorías perso- nales» y teorías científicas, o de los datos empíricos aportados por investigaciones como las de Driver y Erickson (1983), Engel Clough y Driver (1986), Jiménez Aleixandre (1990), Luffiego, Bastida, Ramos y Soto (1991) o Serrano (1993), constatando la existencia de marcos o esquemas conceptuales, a través de los cuales se relacionan diversas ideas específicas.

Por nuestra parte, en investigaciones realizadas para averiguar las ideas de las alumnas y los alumnos sobre los procesos de nutrición humana, también hemos liamado la atención con respecto a esta misma cuestión, señalando que, además de concepciones puntuales (escasamente relacionadas entre sí), es posible encontrar esquemas conceptuales más amplios, a través de los cuales se relacionan e integran diversos conocimientos relativos a un mismo proceso. Asi lo hemos puesto de manifiesto al referirnos, por ejempio, a la digestión o a la respiración humana (Banet y Núñez, 1989, 1990).

En un trabajo más reciente (Núñez, 1994), dirigiđo a profundizar en el conocimiento de las ideas de los alumnos y alumnas sobre nutrición, se ha podido constatar la existencia de un tercer nivel de organización de dichas ideas, que hemos denominado modelos concep- 
tuales. De forma similar a lo que otros autores describen como teorías personales, marcos o esquemas conceptuales, entendemos por modelos conceptuales el conjunto de concepciones a través de las cuales se establecen diversos tipos de relación entre los procesos que intervienen en la nutrición humana y se caracterizan por los siguientes rasgos:

a) Incluyen concepciones relacionadas entre sí, que se estructuran en forma piramidal; es decir, están compuestos de una serie de nociones específicas organizadas jerárquicamente.

b) Presentan cierto grado de coherencia interna, que se comprueba a partir de la regularidad que se observa en las explicaciones de los alumnos.

c) Constituyen la base en que se apoyan las y los estudiantes para explicar los diversos aspectos relacionados con la nutrición, y suponen el punto de partida para el aprendizaje de nuevos contenidos referidos a dichos procesos.

d) Permiten deđucir las dificultades que encontrarán las alumnas y los alumnos en el aprendizaje de estas nociones, constituyendo -al mismo tiempo- un elemento importante de cara a la planificación y desarrollo de propuestas didácticas.

A través de la mencionada investigación, se constata la existencia de dos tipos de modelos, con distinto grado de generalidad: modelos parciales, indicativos de las relaciones que establecen las alumnas y los alumnos entre digestión, respiración y el sistema circulatorio, y modelos globales, los cuales representan su visión general sobre la nutrición humana en su conjunto. Evidentemente, ambos tipos de modelos están íntimamente relacionados: Ios modelos parciales pueden servir de base para determinar los modelos globales, cuestión sobre la que estamos trabajando en estos momentos.

A lo largo de este artículo describimos las características de los modelos parciales de nutrición, haciendo referencia a su representatividad en una muestra amplia de estudiantes pertenecientes a diferentes niveles educativos. Del análisis de los resultados se desprende una serie de conclusiones en relación con el modo en que se organizan y estructuran las concepciones de los alumnos y con respecto a la enseñanza/aprendizaje de estas nociones en los niveles básicos de educación.

\section{METODOLOGÍA DE LA INVESTIGACIÓN}

La investigación ha sido realizada con una muestra de estudiantes ( $\mathrm{N}=444$ ) pertenecientes a aquellos niveles educativos no universitarios en los cuales se estudian estas nociones: $6^{\circ}$ de EGB (159), $8^{\circ}$ de EGB (167), $1^{\circ}$ de BUP (72) y $3^{\circ}$ de BUP (46).

Teniendo en cuenta la diversidad de la población, el grado de diferenciación conceptual con que se ha efec- tuado la exploración (Fig. 1), hace referencia a aquellos aspectos que consideramos esenciales -en cualquiera de los niveles- para la comprensión de la nutrición como un conjunto de procesos íntimamente relacionados. Fundamentalmente, nos centramos en averiguar hasta qué punto se comprende el papel del sistema circulatorio en la nutrición humana, a un doble nivel:

a) Relaciones digestión/circulación: las sustancias nutritivas resultantes de la digestion de los alimentos son utilizadas por los órganos (células) de nuestro cuerpo. EI sistema circulatorio es el encargado de transportarlas.

b) Relaciones respiración/circulación: el oxf́geno recogido en los pulmones es llevado por la sangre a los diferentes órganos (células). Del mismo modo, la sangre recoge dióxido de carbono procedente de los órganos (células) para ser eliminado a través de los pulmones.

La recogida de información se basa en la administración de un cuestionario, cuya elaboración consta de cuatro fases (Treagust, 1988; Anderson, Sheldon y Dubay, 1990; Seymour y Longden, 1991):

1) ensayo previo, mediante la realización de entrevistas individuales a un grupo reducido de 8-10 alumnos/as por cada nivel, de cara a confeccionar un cuestionario adecuado a sus conocimientos;

2) estudio piloto, consistente en la administración del cuestionario provisional (elaborado a partir de la fase anterior) a un grupo de alumnos/as pertenecientes a los diferentes niveles objeto de estudio;

3) reformulación del cuestionario inicial a partir de los datos suministrados por el estudio piloto y la revisión realizada por compañeros investigadores y los profesores de ciencias correspondientes a la muestra utilizada en la fase anterior;

4) administración del cuestionario definitivo a la muestra objeto de nuestra exploración.

El cuestionario utilizado contiene 19 cuestiones de diversos tipos: respuesta abierta, elección múltiple y doble elección «respuesta-razón» (Haslam y Treagust, 1987). Para evitar el carácter aleatorio de ciertas respuestas ola información restringida que se obtiene de alguna de ellas, se utilizan diferentes preguntas para incidir en un mismo aspecto (como propone Yarroch, 1991), y se complementan las cuestiones más complejas con una escala de fiabilidad, para que las y los estudiantes expresen el grado de certeza en sus afirmaciones. (En el anexo I se muestran, a modo de ejemplo, algunas preguntas incluidas en el cuestionario, que por su extensión no reproducimos integramente. No obstante, queda a disposición de quien lo solicite.)

En sintesis, la exploración se ha centrado sobre los siguientes aspectos:

- Digestión de los alimentos: Se plantea una situación problemática (digestión de la leche), a partir de la 
cual se pregunta qué típo de sustancias obtenemos mediante dicho proceso (complejas, sencillas, sustancias buenas...) y dónde se absorben.

- Relaciones entre el proceso digestivo y la circulación de la sangre: Se utilizan tres cuestiones. Las dos primeras consisten en situaciones problemáticas: qué sucede cuando estamos sin tomar alimentos durante dos o tres días; si las sustancias que transporta la sangre salen de Ios vasos sanguíneos cuándo llegan a los músculos. En la tercera se pregunta sobre el transporte de los nutrientes a los órganos.

- Respiración y relación entre dicho proceso y la circulación de la sangre: Se indaga en torno a la composición de los gases inspirado y espirado; a la relaciones entre el sistema circulatorio y el transporte de los gases respiratorios (por qué respiramos más deprisa después de correr $200 \mathrm{~m}$; qué ocurre cuando estamos buceando y contenemos la respiración o cuando dormimos); al transporte de oxígeno y díxxido de carbono (por medio de dibujos esquemáticos y preguntas de elección múltiple); y cuestiones referidas a la necesidad de oxígeno por los distintos organos.
- Estructura celular de nuestro cuerpo: Para ello, se incluyen tres preguntas. La primera está relacionada con la noción general que poseen los estudiantes sobre la célula y la relación entre éstas y los órganos; en la segunda se les facilita una relación de órganos y se les pide que señalen si están formados por células; la tercera incide en la cuestión de las sustancias necesarias para que las células realicen sus funciones.

El análisis de las respuestas de las alumnas y los alumnos se basa en la regularidad y coherencia que presentan a través de los diferentes instrumentos empleados (diversos ítems del cuestionario y entrevistas individuales), tomando como referencia el nivel conceptual establecido en la figura 1. En resumen consta de las siguientes fases:

1) Una vez tabulados los datos correspondientes a cada pregunta del cuestionario, establecemos una serie de concepciones específicas y esquemas conceptuales sobre aspectos parciales de los procesos objeto de estudio. Para ello, contrastamos la correlación existente entre las respuestas que dan las y los estudiantes a aquellas cuestiones que se refieren a los mismos aspectos (p.e., sus-

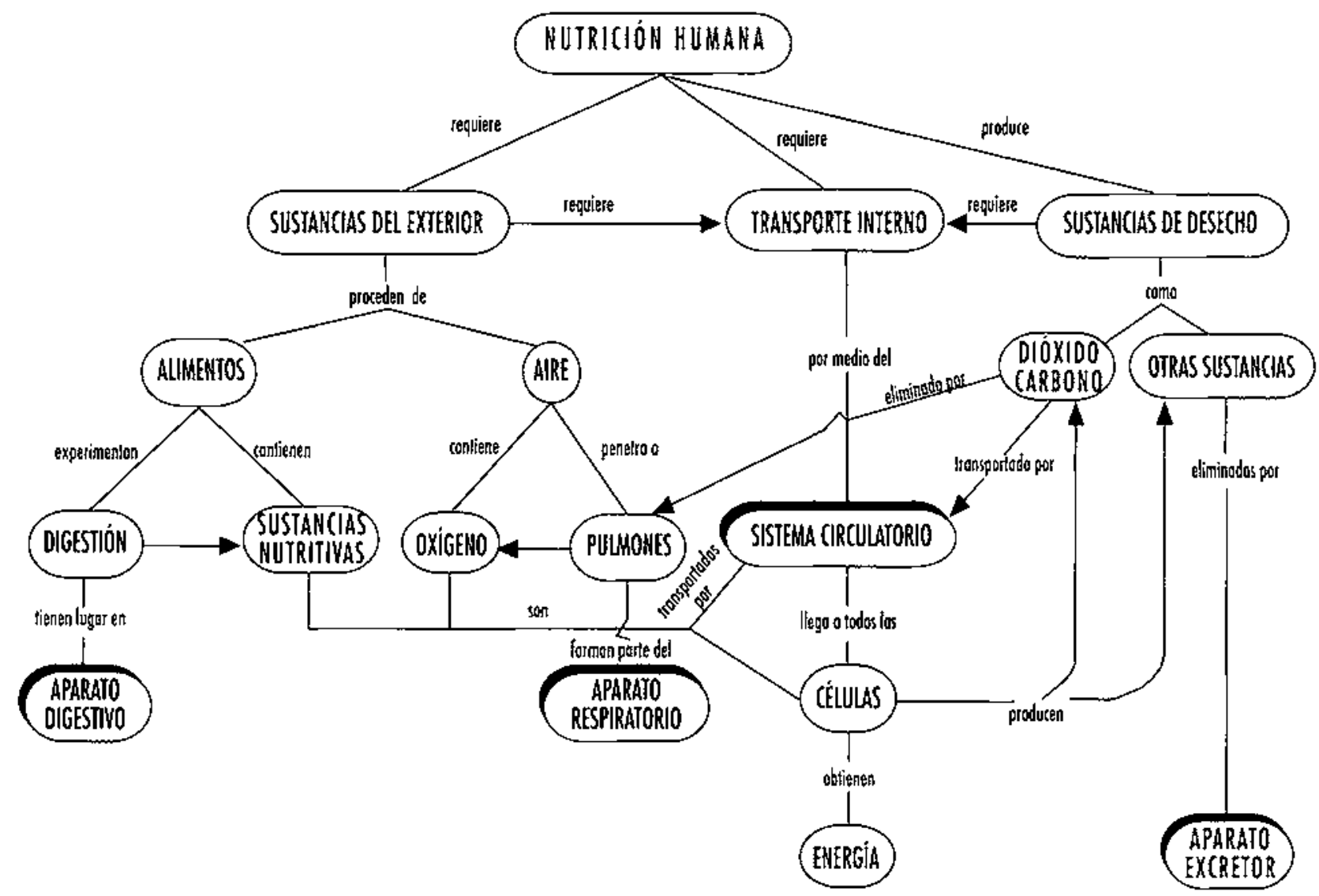


tancias que se obtienen mediante la digestión, sustancias que transporta la sangre, destino de dichas sustancias...).

2) Definición de los modelos conceptuales que subyacen al conjunto de concepciones puntuales y esquemas conceptuales relativos a las relaciones existentes entre algunos procesos de nutrición (digestión/circulación, respiración/circulación).

3) Identificación de los modelos conceptuales correspondientes a cada uno de los alumnos y alumnas pertenecientes a la muestra utilizada. En aquellos casos en que sus concepciones parecían incompletas o contradictorias, recurríamos a la realización de entrevistas individuales para su clarificación.

\section{DESCRIPCIÓN DE LOS MODELOS CONCEPTUALES Y DISCUSIÓN DE LOS RESUITAADOS}

Al margen del diferente grado de profundidad con que se estudia la nutrición en los niveles educativos explorados, que lógicamente se refleja en la riqueza de las concepciones de los alumnos y las alumnas, la investigación ha puesto de manifiesto la existencia de una serie de modelos conceptuales comunes a todos ellos, aunque extendidos de manera desigual. Los diversos modelos encontrados (referidos tanto a las relaciones digestión/ circulación como a las relaciones respiración/circulación) tienen un carácter progresivo y están ordenados en función de que scan más o menos acordes con el conocimicnto científico: no relacionados, parcialmente relacionados y relacionados.

\section{A. Modelos de relación entre digestión y circulación sanguínea}

Encontramos un total de seis modelos, agrupados en torno a las tres categorías antes mencionadas.

\section{Modelos no relacionados}

Clasificamos en este grupo aquellas concepciones caracterizadas por la falta de relaciones adecuadas entre el proceso digestivo y la circulación sanguínea, en el ámbito de la nutrición humana. En esta categoría encontramos dos modelos, en función de si las alumnas y los alumnos mencionan o no el papel de la sangre en el transporte de sustancias nutritivas procedentes de los alimentos.

\section{Modelo I}

De las explicaciones de las y los estudiantes encuadrados en este modelo (Fig. 2) se deduce que, para ellos, las sustancias que se obtienen como consecuencia de la digestión đe los alimentos (proceso que entienden como separación de las sustancias buenas y malas que éstos contienen) no son recogidas por la sangre, sino que recorren el tubo digestivo y finalmente son eliminadas. Por tanto, además de representar una visión incompleta de la digestión, en este modelo se simplifica la nutrición considerando que el simple tránsito de sustancias a través del aparato digestivo garantiza la supervivencia humana.

\section{Modelo 2}

La progresión de este modelo con respecto al anterior estriba en que se admite que la sangre transporta las sustancias nutritivas obtenidas durante la digestión (en ocasiones, "sustancias buenas»), atnque se afirma que éstas no salen de los vasos sanguíneos y, por tanto, se desconoce su destino y el modo en que son utilizadas. Así se deduce de explicaciones como las siguientes:

Profesor. Una vez que la sangre llega, por ejemplo, a los músculos, ¿qué ocurre con las sustancias nutritivas que transporta?

Alumno ( $6^{\circ}$ de EGB). Yo creo que esas sustancias van recorriendo todo el cuerpo.

P. ¿Pero salen de los vasos sanguíneos (venas, arterias...) o no?

A. No, la sangre pasa por los músculos yotras partes del cuerpo, pero no pueden salir porque entonces no nos alimentarian.

Esta forma de entender el papel de transporte de la sangre estaría relacionada con la atribución al propio cuerpo de una serie de funciones «mágicas» (para nutrirnos es suficiente con que las sustancias nutritivas vayan circulando con la sangre), que -en definitiva - resultan exponente de la escasa comprensión de aspectos esenciales de los procesos de nutrición humana.

Los modelos no relacionados se encuentran ampliamentc extendidos en $6^{\circ}$ de EGB ( $57 \%$ globalmente), mientras que resultan minoritarios en $8^{\circ}$ de EGB y $1^{\circ}$ de BUP ( $25 \%$ y $11 \%$, respectivamente) y -obviamente- no se dan en $3^{\circ}$ de BUP (Fig. 6). Estos datos, parecen confirmar una idea apuntada por autoras como Gellert (1962), Nagy (1953) o Carey (1985), en el sentido de que, para los alumnos y las alumnas de estas edades, el funcionamiento del cuerpo humano tiene un carácter finalista: cada órgano/aparato realiza una función específica, independientemente de los demás. De ahí que la participación del aparato digestivo y el sistema circulatorio en la nutrición humana no se relacionen correctamente.

Por otra parte, la existencia de dichos modelos indica que, a pesar del grado de detalle con que se estudian estas nociones en los niveles básicos de enseñanza (quizás, precisamente por eso), un gran número de estudiantes elabora concepciones muy superficiales al simplificar la nueva información para acomodarla a su modo particular de percibir la realidad. 


\section{Modelos parcialmente relacionados}

El conjunto de concepciones correspondientes a esta categoría de modelos, intermedia entre la anterior y los que consideramos correctos desde el punto de vista de la ciencia, se caracteriza por un mayor reconocimiento del papel de la sangre como medio de transporte de nutrientes, aunque tampoco -en este caso-se alude claramente a las células como el lugar en que son utilizados. Las respuestas de los alumnos permiten diferenciar dos modelos (Fig. 3):

\section{Modelo 3.1}

A través de las explicaciones que dan los alumnos a las diferentes preguntas formuladas en relación con estos aspectos, se constata su creencia de que los procesos relacionados con la nutrición tienen lugar en los diferentes órganos de nuestro cuerpo:

Profesor. Supongamos que estamos sin tomar alimentos durante dos o tres días. ¿Qué ocurre con las sustancias nutritivas que lleva la sangre?
Alumna ( $8^{\circ}$ de EGB). Se acabarian.

P. ¿Por qué?

A. Porque la sangre las lleva a los órganos de nuestro cuerpo y allí se consumen.

P. ¿Cómo utilizan los órganos (por ejemplo, los músculos) esas sustancias?

A. Las gastan para funcionar, pero no se cómo.

P. ¿Los músculos están formados por células?

A. Si.

P. Las sustancias nutritivas que obtenemos de los altmentos, ¿son utilizadas por las células?

A. No, las células se alimentan por si solas.

De ahí que sitúen el destino de las sustancias nutritivas en dichas estructuras, desconociendo las relaciones existentes entre los niveles macroscópico (órganos) y microscópico (células).

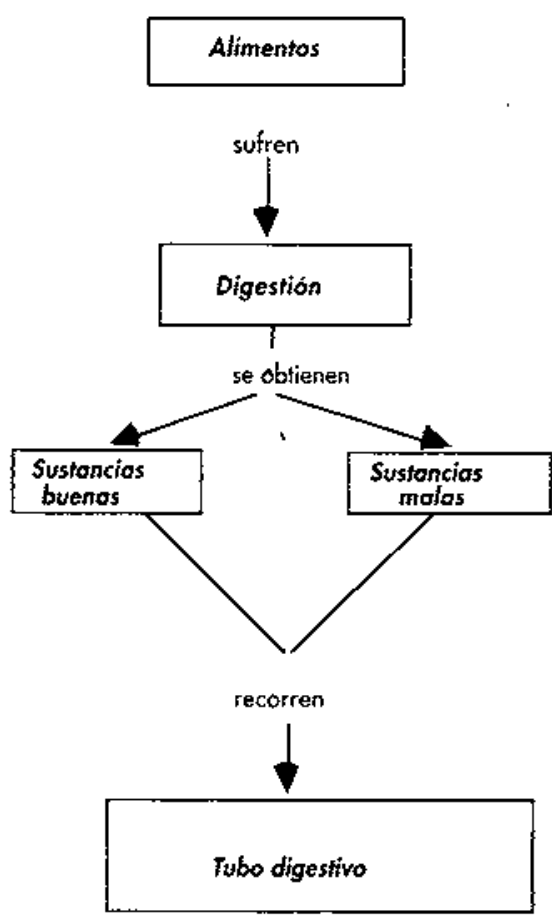

Modelo I

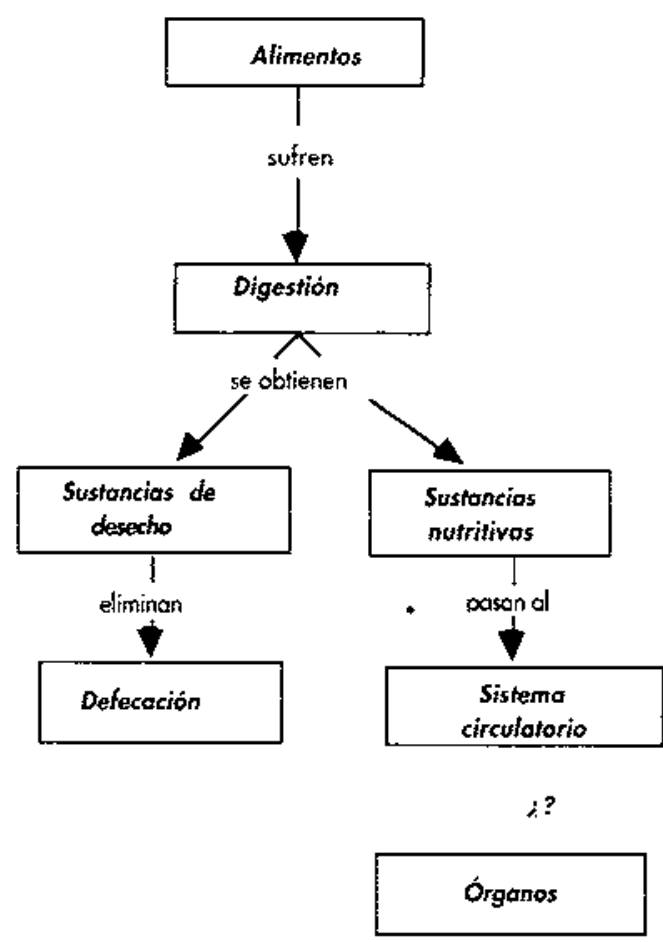

Modelo 2 
Figura 3

Modelos digestión/circulación (parcialmente relacionados).

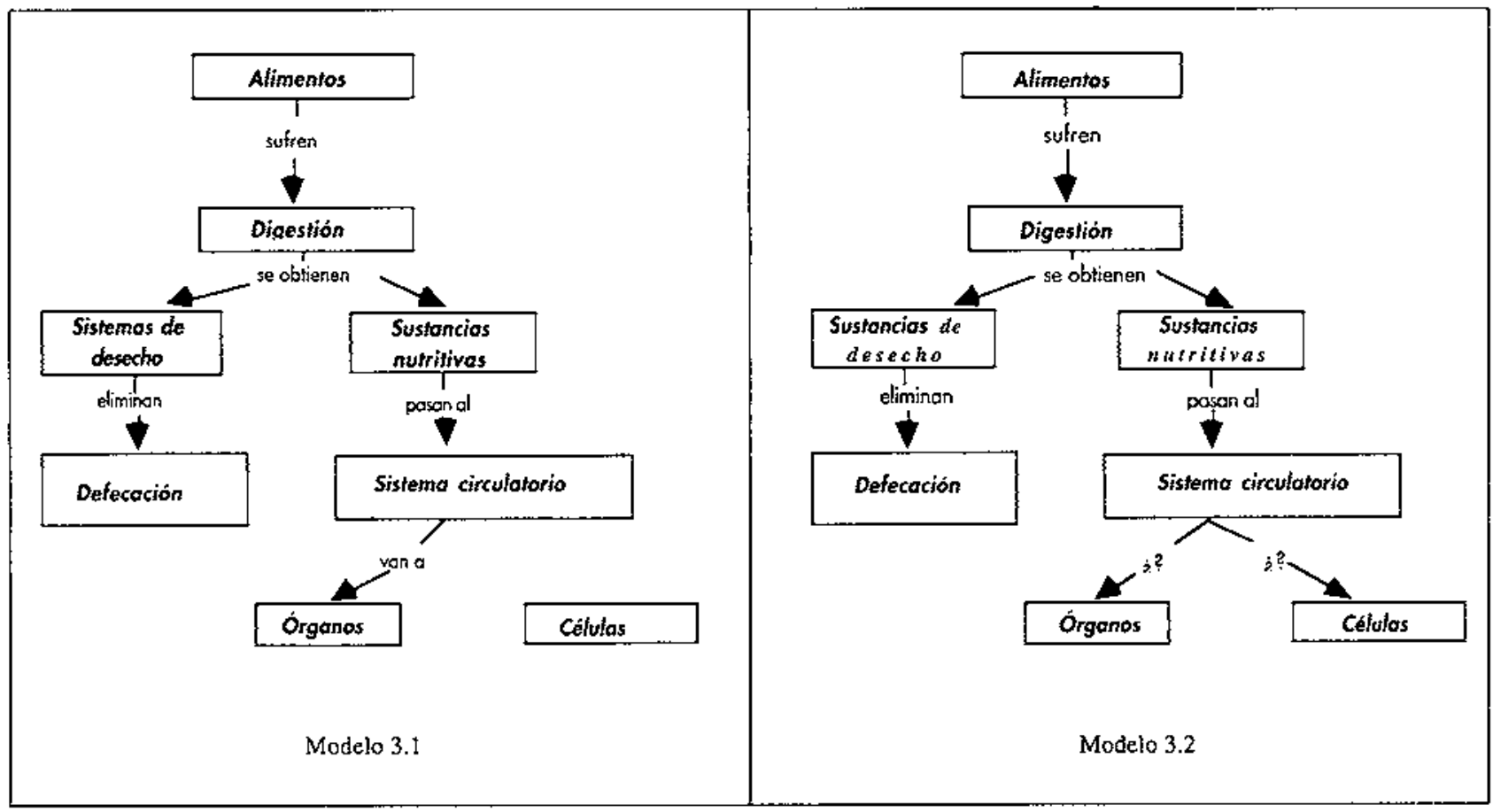

\section{Modelo 3.2}

Aunque, en cierta medida, las concepciones correspondientes a este modelo suponen un avance con respecto al anterior (implican aceptar que las sustancias nutritivas puedan ser utilizadas en las células), presentan una serie de contradiciones que justifican su inclusión en la categoría de modelos parcialmente relacionados: considerar que el destino de las sustancias nutritivas son, en unos casos, los órganos y en otros las células; creer que unos organos estân formados por células y otros no; o afirmar que se produce un doble intercambio de sustancias nutritivas entre la sangre y las células.

A la vista de los resultados (Fig. 6) podemos afirmar que los modelos parcialmente relacionados se detectan en todos Ios niveles educativos investigados, aunque con diferente grado de representatividad y significación. Mientras que -hasta cierto punto- nos parece lógico que en $6^{\circ}$ de FGB el máximo grado de relación entre digestión y circulación se sitúe en el modelo 3.1 (37\% del total), no pensamos to mismo con respecto al porcentaje de estudiantes de $8^{\circ}$ de EGB y $1^{\circ}$ de BUP encuadrados en el mismo (14\% y $18 \%)$, puesto que en estos niveles se suele ampliar el estudio de la nutrición haciendo referencia a algunos procesos celulares como la obtención de energía o el crecimiento.

Aunque la existencia de cierta "controversia» órganoscétulas, en cuanto al destino de las sustancias nutritivas (modelo 3.2 ), no se halla muy extendida ( $10 \%$ en $8^{\circ}$,
$18 \%$ en $1^{\circ}$ de BUP y $22 \%$ en $3^{\circ}$ de BUP), a nuestro juicio, pone de manifiesto que la entrada de nuevas informaciones al estudiar estas nociones se ha traducido en un aumento de vocabulario que, en la mayoría de los casos, no ha modificado las concepciones iniciales de las y los estudiantes, al no producirse un aprendizaje significativo de los nuevos contenidos. De sus explicaciones deducimos la existencia de un obstáculo epistemológico, señalado por autores/as como Dreyfus y Jungwirth (1988), Gagliardi (1988) o Caballer y Jiménez (1992): el desconocimiento de la estructura y funcionamiento celular de nuestro cuerpo y, en particular, de las relaciones entre los procesos a nivel anatómico y sus referentes a nivel celular.

\section{Modelos relacionados}

Constituyen una relación adecuada entre digestión y circulación, al nivel que se ha realizado la investigación (Fig. 1), independientemente de que en los cursos más avanzados se posea un mejor conocimiento de las mismas. Dentro de esta categoría se pueden diferenciar dos modelos, en función de cual sea el destino de las sustancias nutritivas (Fig. 4):

\section{Modelo 4.1}

Las concepciones correspondientes a este modelo coinciden en señalar el papel de la sangre como medio de 
Figura 4

Modelos digestión/circulación (relacionados).

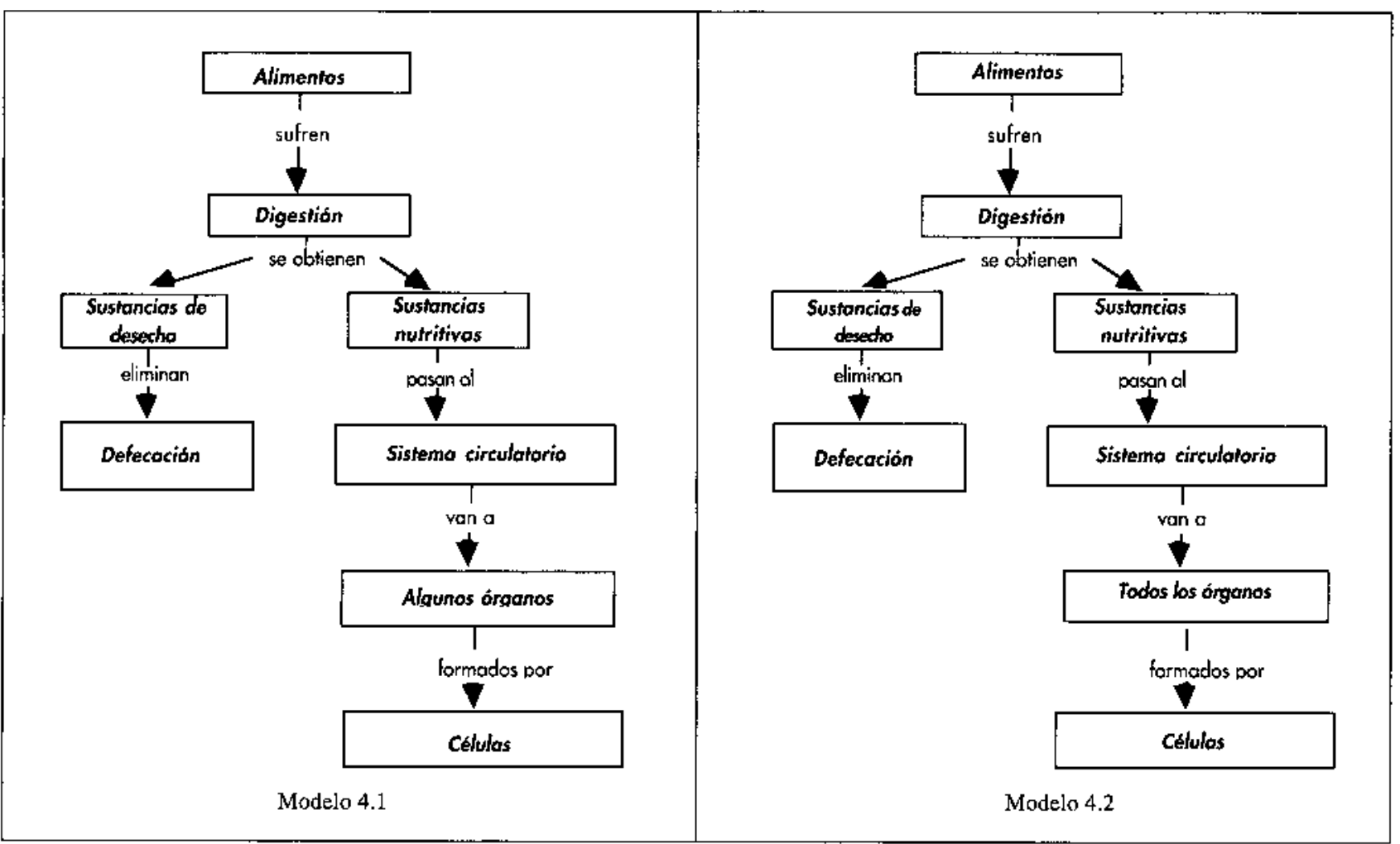

relación entre procesos: recoge las sustancias nutritivas procedentes de la digestión y las transporta hacia las células, en donde serán utilizadas para la realización de determinadas funciones (crecer, estar fuertes...).

Probablemente como consecuencia de un conocimiento incompleto de la estructura y organización celular de nuestro cuerpo, los alumnos y las alumnas encuadrados en este modelo afirman que determinados órganos (pulmones, corazón, huesos...) no necesitan nutrientes para realizar sus funciones (Fig. 5).

\section{Modelo 4.2}

Supone cierto grado de progresión con respecto al modelo anterior, al entender que todos los órganos están formados por células y que los nutrientes obtenidos mediante la digestión de los alimentos son necesarios para el desarrollo de procesos que están relacionados con la nutrición humana, los cuales son imprescindibles -a su vez-para el funcionamiento de los órganos.

Como cabría esperar a priori, el mayor grado de relación entre los procesos objeto de análisis se produce en $3^{\circ} \mathrm{de}$ BUP (70\% globalmente) y, en menor medida, en $8^{\circ}$ de EGB $(43 \%)$ y $1^{\circ}$ de BUP $(49 \%)$, tal y como se desprende de la figura 6. Los resultados en estos últimos niveles pueden ser interpretados en dos sentidos: si bien tienen de positivo que alrededor de la mitad de la muestra posean un conocimiento adecuado de las relaciones digestion/circulación, por contra, muestran que no se produce progresión conceptual entre ambos niveles, a pesar del estudio de la nutrición humana incluyendo lecciones específicas sobre el conocimiento de la célula (estructura y funciones).

Abundando en el análisis de estos datos, constatamos que es significativamente menor el porcentaje de estudiantes encuadrados en el modelo más avanzado, como consecuencia de las dudas existentes en torno a si el destino de las sustancias nutritivas que transporta la sangre son todos los órganos o una parte de ellos. En ese sentido, resulta esclarecedor comprobar (Fig. 5) cómo las mayores discrepancias se producen en relación con una serie de órganos que realizan funciones muy específicas (pulmones, corazón...) o bien con aquéllos que aparentemente no realizan ninguna actividad (huesos), admitiendo -por el contrario- que los órganos más relacionados con el movimiento corporal (músculos), o que se consideran más importantes para el organismo (cerebro), precisan sustancias nutritivas para su funcionamiento. 
Figura 5

Órganos que no necesitan sustancias nutritivas

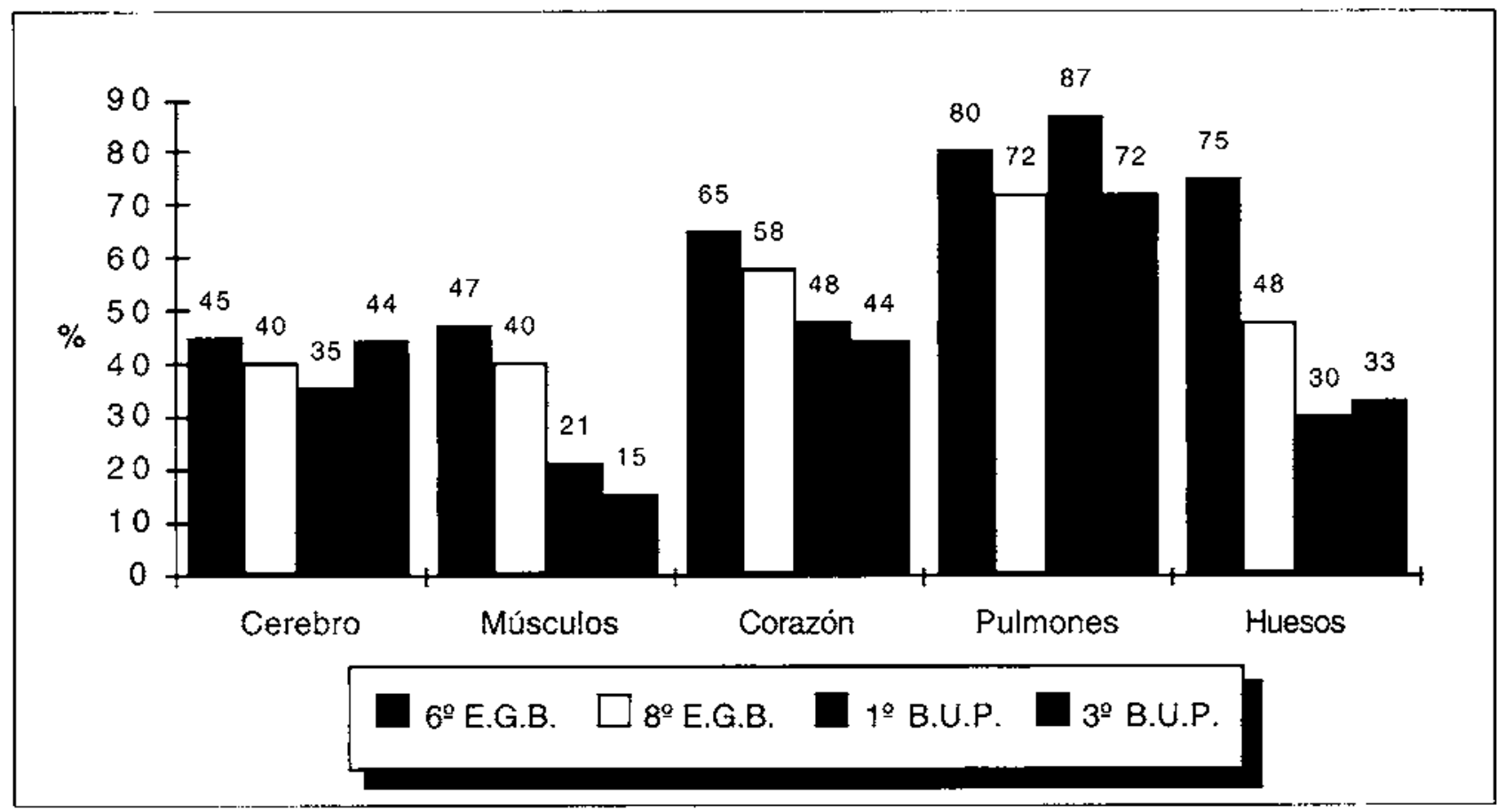

Figura 6

Modelos digestión/circulación (resultados).

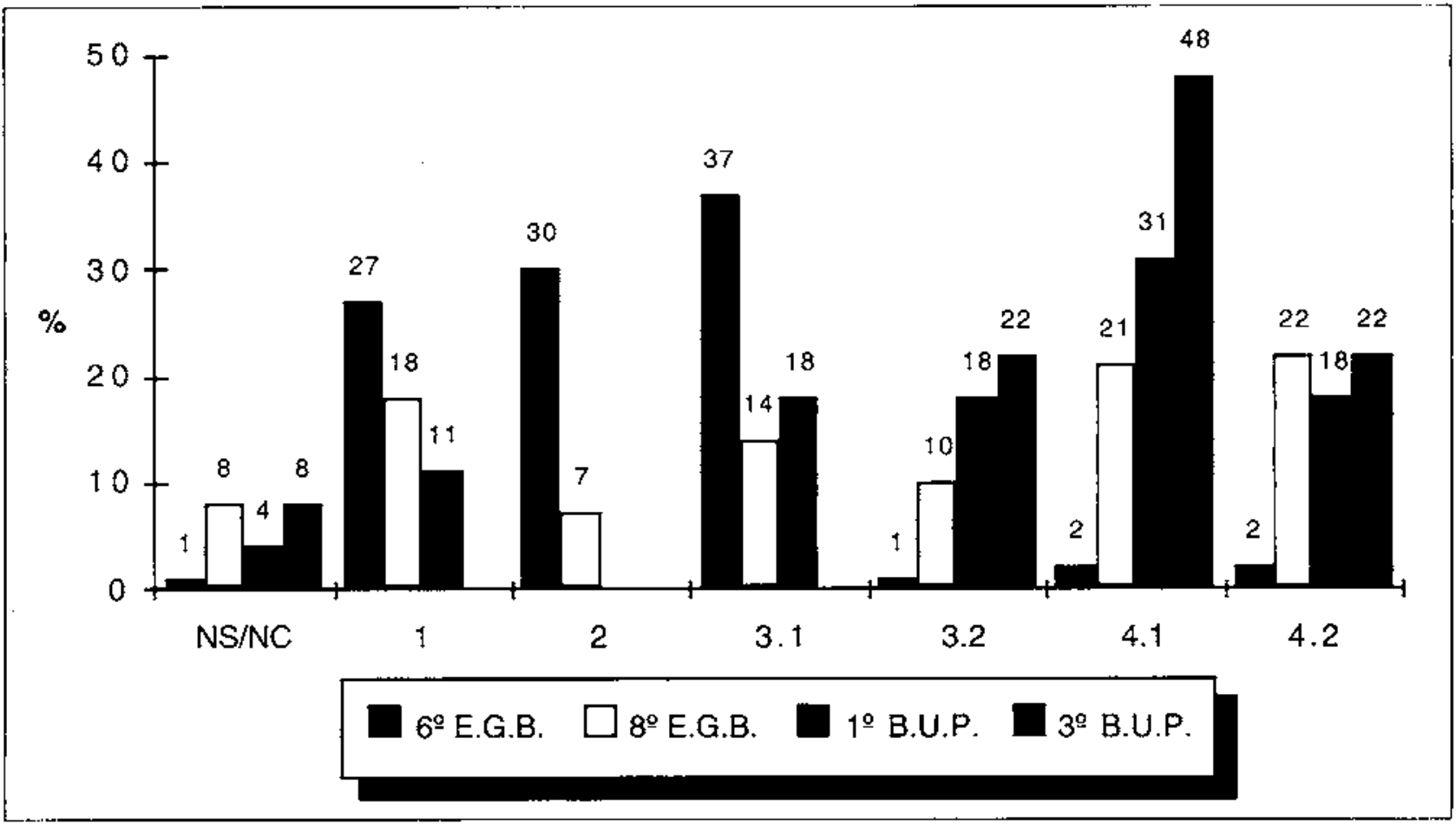


Así como en $8^{\circ}$ de EGB y $1^{\circ}$ de BUP estos últimos resultados, coincidentes, por otra parte, con trabajos como los de Caballer y Jiménez (1992, 1993) sobre la célula, pueden explicarse por la falta de consolidacion de determinados conceptos, no pensamos lo mismo con respecto a los de $3^{\circ}$ de BUP, teniendo en cuenta que se trata de estudiantes que han cursado biología y química con un elevado grado de diferenciación conceptual.

\section{Modelos de relación entre respiración y cịculación sanguínea}

Tal vez como consecuencia de las dificultades que plantea la comprension del proceso respiratorio y su relación con la circulación sanguínea, la investigación ha puesto de manifiesto la existencia de una amplia gama de modelos conceptuales (nueve en total) que, no obstante, se pucden agrupar en torno a las tres categorías establecidas en el apartado anterior: no relacionados, parcialmente relacionados y relacionados.

\section{Modelos no relacionados}

Ios cinco modelos pertenecientes a esta categoría (Fig. 7 y 8) tienen en común no identificar la respiración como un proceso cclular $y$, por consiguiente, no relacionar correctamente el papel de la sangre como medio de transporte del oxígeno desde los pulmones a las cólulas y del dióxido de carbono desde éstas hasta cl aparato respiratorio. Dichos modelos, clasificados de menor a mayor grado de relación y diferenciación conceptual, presentan las siguientes características:

\section{Modelo I}

Supone considerar la respiración como un proceso que tiene lugar en los pulmones, mediante la entrada de oxígeno y la salida de dióxido de carbono. Ia existencia de este modelo incluye concepciones como las siguientes: a) el airc que entra a los pulmones contiene solamente oxígeno, gas necesario para vivir; $b$ ) el aire que espiramos sólo contiene dióxido de carbono (que se origina a partir del oxígeno o cs el mismo que entró al inspirar); $c$ ) la sangre no transporta oxígeno ni dióxido de carbono (este último es tóxico).

\section{Modelo 2.I}

Aunque los alumnos y alumnas encuadrados en este modelo admiten que la sangre pueda transportar oxígeno, entienden la respiración como un proceso principal-

Figura 7

Modelos respiracion/circulación (no relacionados 1 ).

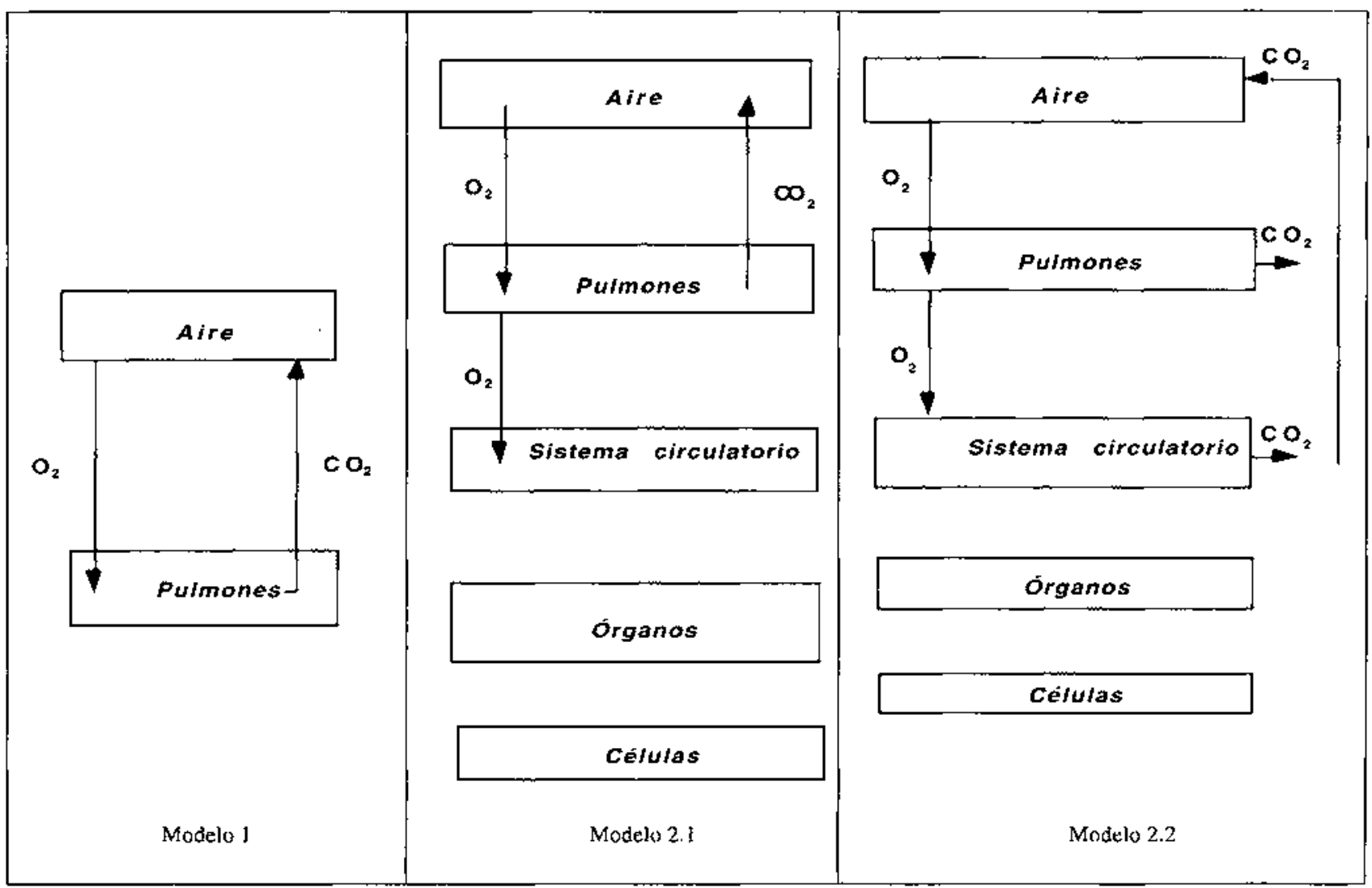


Figura 8

Modelos respiración/circulación (no relacionados 2) y parcialmente relacionados.

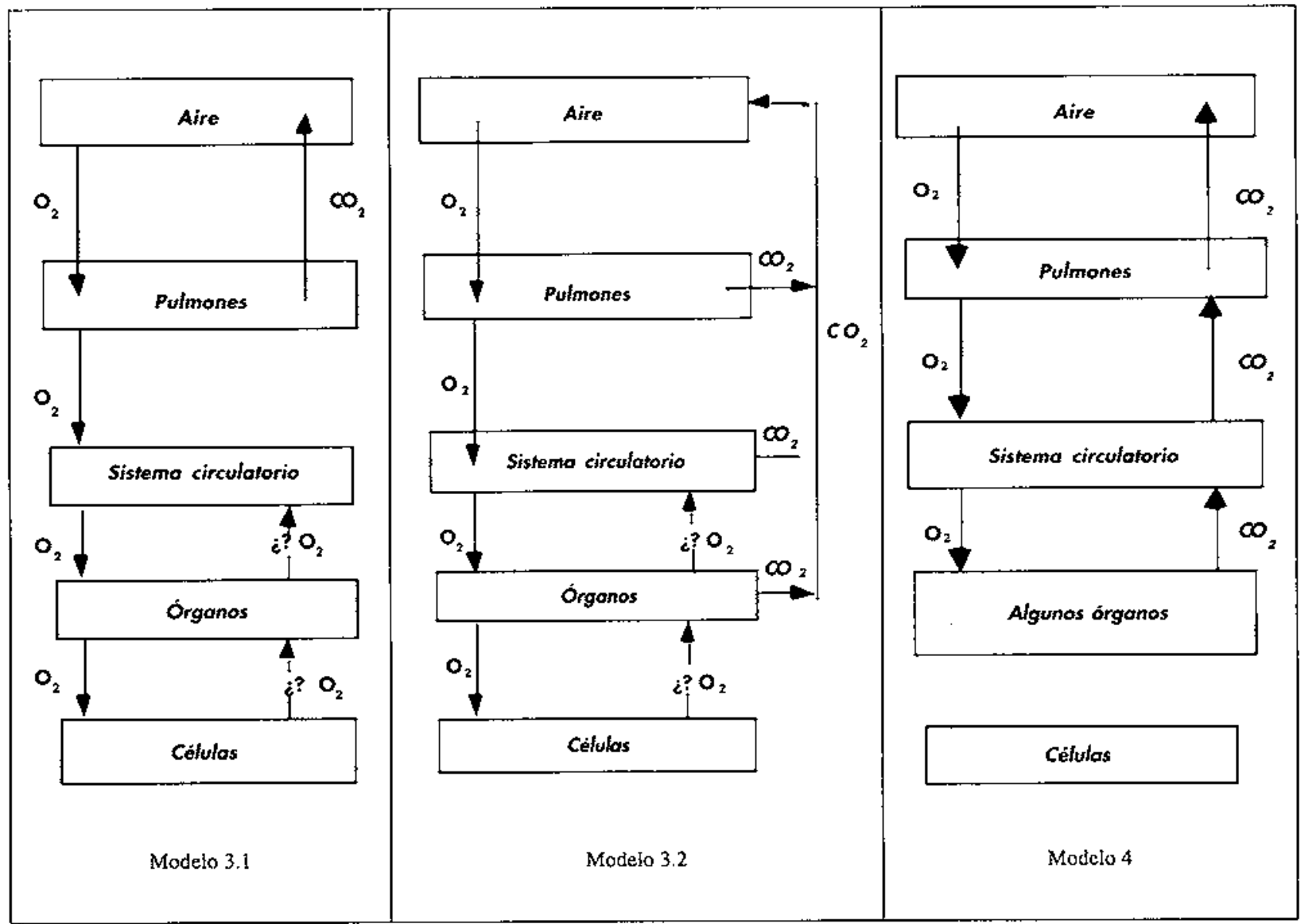

mente pulmonar y atribuyen al sistema circulatorio un papel secundario: a) la sangre transporta oxígeno pero éste no sale de los vasos sanguíneos (sblo circula), por tanto, se desconoce su destino y utilidad; b) la sangre no transporta dióxido de carbono, ya que dicho gas se origina en los pulmones.

Esta aparente contradicción no es tal para las y los estudiantes, como se comprueba a través de suss explicaciones:

Profesor. ¿En qué consiste la respiración?

Alumno ( $8^{\circ}$ de EGB). Cuando inspiramos, tomamos oxigeno y, cuando espiramos, expulsamos dióxido de carbono.

P. ¿Para qué necesitamos el oxigeno del aire?

A. Lo necesitan los pulmones para respirar. Si no fuese así nos moririamos.

P. Además de los pulmones, ¿qué otros órganos necesitan oxigeno?
A. No lo sé. Creo que solamente lo necesitan los pulmones.

P. Antes me has dicho que la sangre transporta sustancias nutritivas por todo el cuerpo; ¿lleva también oxigeno y dióxido de carbono?

A. Oxigeno sí, pero dióxido de carbono no porque como es tóxico nos moriríamos.

P. Cuando la sangre llega a órganos como los músculos, ¿qué ocurre con el oxígeno que transporta?

A. No lo sé.

P. Quiero decir, si el oxigeno sale de las arterias y es utilizado en los músculos.

A. No, el oxigeno recorre el cuerpo pero no puede salir de las venas.

P. Entonces, ¿como es posible que los músculos funcionen?

A. Porque tomamos alimentos como la leche o la came, que nos dan mucha energía. 
Figura 9

Modelos respiración/circulación (relacionados).

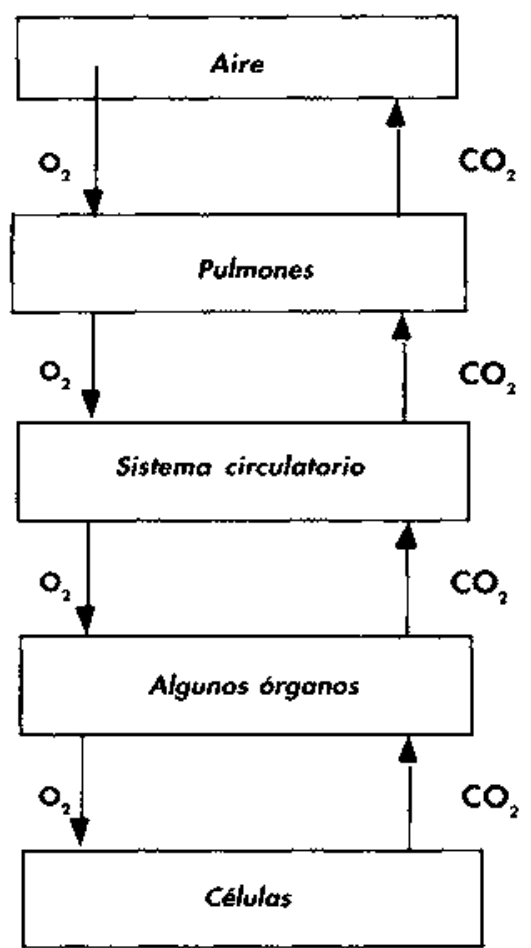

Modelo S.1

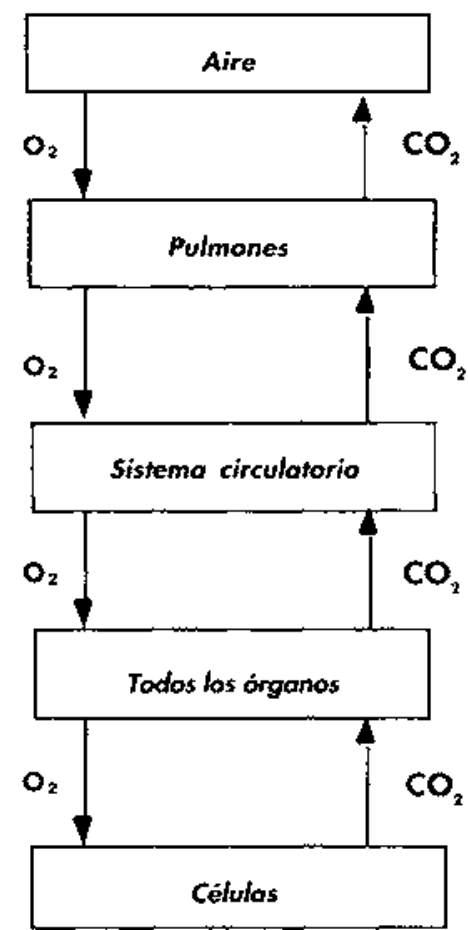

Modelo 5.2

\section{Modelo 2.2}

Se diferencia del modelo anterior en cuanto al origen del dióxidio de carbono: aunque la mayor parte de dicho gas se produce en los pulmones (o es el mismo que entró al inspirar), se admite que la sangre lo transporta. No obstante, ello no implica entender su relación con los procesos de nutricion (se afirma que se origina en los órganos, el corazón o la propia sangre), sino que - probablemente-constituya un ejemplo de la «distorsión” de nuevas informaciones que no han sido comprendidas suficientemente.

\section{Modelo 3.1}

Junto con el modelo siguiente, supone un importante avance con respecto a los anteriores. En él se considera que el oxígeno que transporta la sangre sale de los vasos sanguíneos y va a las células. Sin embargo, consideramos que no se establecen relaciones adecuadas entre respiración y circulación, como se deduce de explicaciones de las y los estudiantes que afirman que el oxígeno es necesario sobre todo en los pulmones o cuando se refieren a un doble intercambio de dicho gas entre la sangre y las células:

Profesor. Supongamos que la sangre que circula por estos vasos sanguíneos (señala la muñeca de un brazo) ha llegado a la mano. ¿Qué sustancias de las que transporta la sangre son necesarias para el funcionamiento de los dedos?

Alumna ( $1^{\circ}$ de BUP). Los dedos necesitan glucosa, proteínas, vitaminas y oxígeno.

P. Pero ¿cómo utilizan esas sustancias?

A. En las células.

P. ¿Cómo?

A. Sé que lo estudié el año pasado, pero no me acuerdo.

P. Una vez que las células realizan sus funciones (aunque no te acuerdes cuáles son), ¿se producen algunas sustancias de desecho? 
Figura 10

Organos que no necesitan oxígeno.

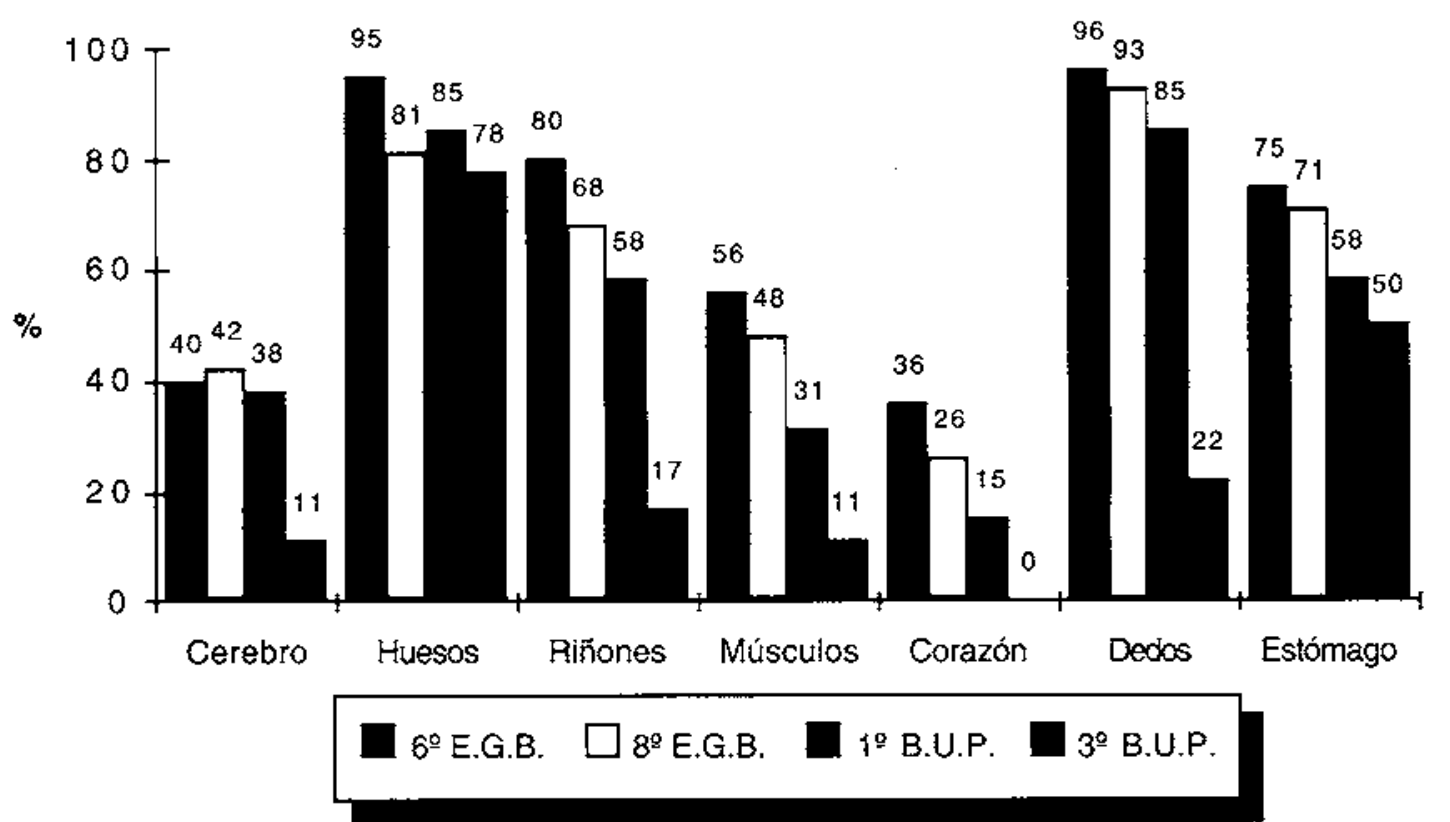

Figura 1 i

Modelos respiracion/circulación (resultados).

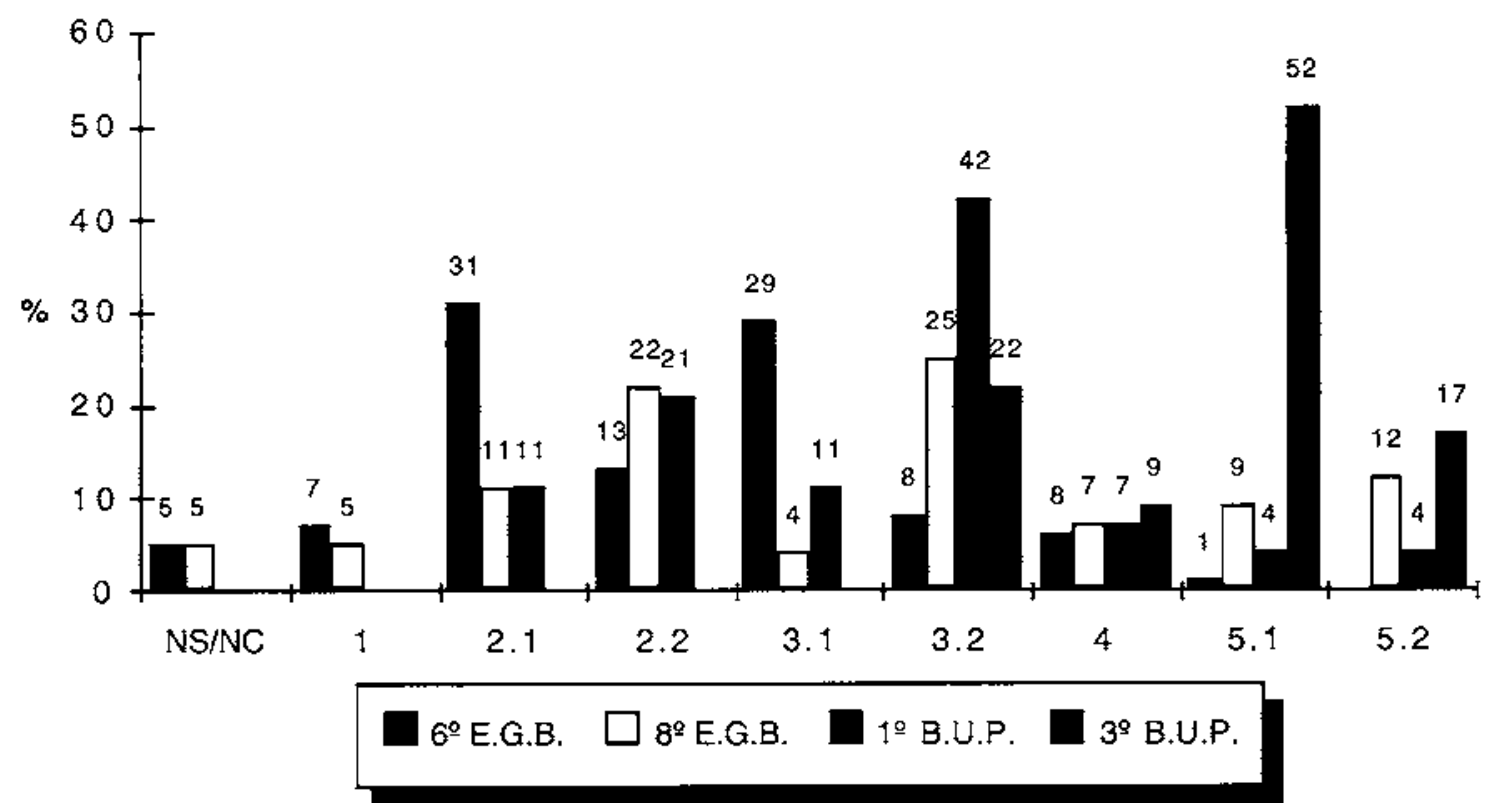




\section{A. Sí. Creo que las células producen vitaminas, oxígeno y otros desperdicios que pasan a la sangre para poder- los expulsar. \\ P. ¿No habias dicho que el oxígeno se obtiene en los pulmones?}

A. La mayoría se produce en los pulmones, pero una parte va a las células para que puedan realizar sus funciones.

P. Entonces, ¿en qué quedamos?; ¿el oxígeno pasa de la sangre a las células o de las células a la sangre?

A. El oxigeno va a las células, pero ellas también dan oxígeno a la sangre.

En relación con el đióxiđo de carbono, dichos estudiantes consideran que se origina en los pulmones, por lo que, en conjunto, se trata de un modelo básicamente pulmonar.

\section{Modelo 3.2}

Se diferencia del modelo anterior únicamente en cuanto al origen del dióxido de carbono, admitiendo que, aunque la mayor parte de dicho gas se obtiene en los pulmones, también se produce en los órganos, el corazón o la propia sangre.

Con excepción de $3^{\circ}$ de BUP, una amplia mayoría de alumnos y alumnas pertenecientes a los diferentes niveles educativos explorados (Fig. 11) poseen concepciones encuadrables en lo que hemos denominado modelos no relacionados (considerados globalmente: $92 \%$ en $6^{\circ}$ de EGB, $67 \%$ en $8^{\circ}$ de EGB y $85 \%$ en $1^{\circ}$ de BUP). No obstante, comprobamos ciertas variaciones de unos niveles a otros:

- En $6^{\circ}$ de EGB predominan los modelos menos avanzados, constatándose dos tipos de concepciones: $a$ ) considerar la respiración como un proceso básicamente pulmonar, minimizando el papel de la sangre en el transporte de gases (modelo 2.1.); b) admitir que la sangre transporta oxígeno hacia las células, pero desconociendo su utilidad y afirmar que el dióxido de carbono se origina en los pulmones. En ambos casos se pone de manifiesto que la enseñanza no modifica -sustancialmente- la creencia de que la respiración se realiza en los pulmones, obteniéndose (a lo sumo) nuevas informaciones que no han sido incorporadas al núcleo central de sus concepciones iniciales.

- En $8^{\circ}$ de EGB y $1^{\circ}$ de BUP, con resultados similares, se produce cierta progresión hacia modelos no relacionados más avanzados (3.1 y 3.2 ), aunque subsisten concepciones propias de niveles anteriores (un tercio de alumnos en modelos 2.1 y 2.2). Sin embargo, tampoco en este caso, dichos avances suponen establecer relaciones adecuadas entre respiración y circulación, concretándose -principalmente- en la elaboración de concepciones incompletas (la sangre transporta oxígeno hacia tas células y dióxido de carbono hasta los pulmones) y la aparición de nuevos errores conceptuales (las células producen oxígeno; el dióxido de carbono se origina en los pulmones, algunos órganos o el corazón; etc.).

Estos resultados ponen de manifiesto las dificultades que encuentran las y los estudiantes para comprender los aspectos esenciales del proceso respiratorio y su relación con el sistema circulatorio, cuestión que ha sido señalada en investigaciones como las de Arnaudin y Mintzes (1985), García Zaforas (1991) y Pérez de Fulate (1992). En particular, hay que destacar el escaso aprendizaje que realizan los alumnos y alumnas de $8^{\circ} \mathrm{de}$ EGB y $1^{\circ}$ de BUP, así como la falta de progresión entre dichos niveles, por tratarse de nociones básicas que son abordadas con cierto grado de profundidad.

\section{Modelos parcialmente relacionados}

En este grupo incluimos un modelo cuyas concepciones sobre las relaciones entre respiración y circulación tjenen en común situar el proceso respiratorio en los órganos (Fig. 8): considerar que las sustancias nutritivas y el oxígeno son llevados a los diferentes órganos de nucstro cuerpo, que en ellos son utilizadas para realizar las funciones vitales, y que -como consecuencia de dichos procesos-se obtiene dióxido de carbono. Evidentemente, esta forma de entender la respiración supone una visión incompleta de su relación con la nutrición humana, al desconocer que dicho proceso tiene lugar en las células.

Aunque se trata de un modelo con escasa representación en los niveles investigados, su existencia pone de manifiesto que algunos alumnos y alumnas simplifican los procesos de nutrición (en este caso, las relaciones respiración/circulación) situándolos en los órganos como consecuencia del desconocimiento de la estructura y organización celular del cuerpo humano.

A nuestro juicio, este modelo podría constituir un nivel intermedio adecuado para cursos como $6^{\circ}$ de $\mathrm{FGB}$ e incluso $8^{\circ}$ de EGB, en calidad de paso previo al estudio de los procesos celulares en cursos posteriores. Por el contrario, la introducción prematura de nociones de tanta complejidad en EGB trae como consecuencia la elaboración de concepciones alternativas y una escasa relación entre los procesos objeto de estudio, tal y como acabamos de señalar.

\section{Modelos relacionados}

Se incluyen en este tipo de modelos aquellas concepciones que relacionan correctamente respiración y circulación, al nivel elemental con que se ha planteado esta investigación (Fig. 1). Es decir, hay que entender que el proceso respiratorio tiene lugar en las células y atribuir al sistema circulatorio el papel de transporte de oxígeno desde los pulmones hacia las células, y del dióxido de carbono (producido como desecho en el transcurso de dicho proceso), desde éstas hasta los pulmones. 
Como en el caso de los modelos digestión/circulación, algunas respuestas de los alumnos y las alumnas permiten diferenciar dos submodelos (Fig. 9) en función de que sitúen la respiración en todos los órganos (modelo 5.2) o sólo en una parte de ellos (modelo 5.1).

Solamente encontramos este tipo de modelos en $8^{\circ}$ de EGB, $1^{\circ}$ de BUP y $3^{\circ}$ de BUP (Fig. 11 ). Mientras que en los dos primeros resulta minoritario el porcentaje de alumnos y alumnas que establecen relaciones adecuadas entre respiración y circulación $(21 \%$ y $8 \%$, respectivamente), este tipo de modelos se encuentra ampliamente extendido en $3^{\circ}$ de BUP ( $69 \%$ si consideramos ambos modelos globalmente).

Los resultandos correspondientes a $8^{\circ}$ de EGB y $1^{\circ}$ de BUP confirman argumentaciones realizadas en apartados anteriores, en el sentido de que existe una escasa correspondencia entre las previsiones de la enseñanza (tal y como aparece en el currículo y los libros de texto) y el aprendizaje que logran los alumnos y alumnas.

Por otra parte, aunque los resultados de $3^{\circ}$ de BUP nos parecen lógicos, dado el nivel elemental con que se ha planteado esta investigación, crecmos que deben ser relativizados, por tres razones:

a) Casi una tercera parte de Ia muestra se encuentra en modelos no relacionados (aunque se trata del más avanzado) o parcialmente relacionados. Muestran, por tanto, concepciones alternativas propias de niveles inferiores.

b) Solamente el $17 \%$ del total se agrupa en el modelo relacionado más avanzado (5.2), mientras que un $50 \%$ presenta algunas dudas con respecto al destino del oxígeno que transporta la sangre. Como se desprende de la figura 10, un porcentaje elevado de estudiantes afirma que órganos como los huesos, dedos, riñones o estómago no necesitan oxígeno para desempeñar sus funciones.

c) A través de algunas respuestas de las y los estudiantes, constatamos errores importantes, como desconocer en qué orgánulos celuiares tiene lugar la respiración celular y qué sustancias son necesarias para ello.

Profesor. ¿En qué consiste la respiración?

Alumno. La respiración es un proceso que se produce en todas las células de nuestro cuerpo y nos sirve para obtener energía.

P. ¿En qué partes de la célula tiene lugar?

A. Yo creo que en los ribosomas.

P. Además del oxígeno, ¿qué otras sustancias son necesarias?

A. Por ejemplo, glucosa.

$P$. En los casos en que no se dispone de esta sustancia, ¿qué otros nutrientes pueden ser utilizados?

\section{A. Por ejemplo vitaminas, proteinas y aminoácidos.}

Aunque nuestro propósito no haya sido analizar en profundidad las ideas de estos alumnos, los resultados nos deben hacer reflexionar sobre la idoneidad de los métodos de enseñanza habituales para modificar concepciones erróneas y propiciar aprendizajes significativos.

\section{CONCLUSIONES}

Como hemos puesto de manifiesto a lo largo de este artículo, buena parte de las concepciones de los alumnos y alumnas sobre aspectos específicos de los diferentes procesos implicados en la nutrición humana (digestión, respiración, circulación...) se encuentran organizados en su estructura conceptual, Ilegando a constituir verdaderos esquemas o teorías (modelos conceptuales), a partir de los cuales podemos interpretar dichas concepciones. Por otra parte, si-como hemos señalado-estos modelos suponen el bagaje conceptual con que los estudiantes afrontan el aprendizaje de nuevas nociones, su conocimiento resulta de gran utilidad para la planificación de la enseñanza.

Los resultados obtenidos han puesto de manifiesto el predominio de modelos conceptuales no relacionados o parcialmente relacionados, caracterizadios por no identificar adecuadamente el papel del sistema circulatorio con los procesos de digestión y respiracion. No obstante, encontramos diferencias significativas al comparar la comprensión de las relaciones digestión/circulación con las de respiración/circulación: mientras en el primer grupo se constata cierto conocimiento en la mayorfa de los cursos investigados, Ia comprensión de las relaciones entre respiración y circulación resulta mucho mús problemática, incluso en niveles superiores.

Comparando los datos relativos a los diferentes niveles educativos, podemos afirmar que, si bien se aprecian ciertas diferencias entre $6^{\circ}$ y $8^{\circ}$ de EGB o de $3^{\circ}$ de BUP con el resto de los niveles, no existe progresion importante entre $1^{\circ}$ de BUP y $8^{\circ}$ de EGB (en algunos casos constatamos un retroceso), a pesar de que dichos estudiantes hayan profundizado en el estudio de la nutrición humana. Si además tenemos en cuenta las precisiones realizadas al referirnos a los resultados en $3^{\circ}$ de BUP, podemos concluir que la enseñanza habitual no contribuye, de manera efectiva, a que los alumnos reestructuren sus concepciones iniciales, desarrollando modelos relacionados que sean acordes con el conocimiento cientáfico.

Sin pretender realizar un análisis exhaustivo de las causas que motivan los resultados comentados, podemos señalar una serie de factores que, entre otros, pueden estar en la base de los mismos:

a) De acuerdo con argumentaciones como las de Driver, Guesne y Tiberghien (1985), el razonamiento infantil 
presenta ciertos rasgos diferenciales (pensamiento dirigido por la percepción, enfoque limitado, causalidad lineal y simple, etc.) que, a nuestro juicio, dificultan la elaboración de modelos relacionados. Por otra parte, si como señalan Pozo, Gómez Crespo, Limón y Sanz Serrano (1991), las características de las teorías implícitas de los alumnos y las alumnas difieren claramente del modo en que se estructuran las teorías científicas, parece lógico que la enseñanza habitual no produzca un acercamiento suficiente entre ambas, prevaleciendo las teorías personales que, a lo sumo, sufren pequeñas reestructuraciones que no modifican sustancialmente sus elementos esenciales.

b) El análisis del currículo y los libros de texto (Núñez, 1994) pone de manifiesto que la enseñanza de la nutríción humana adolece de una serie de deficiencias, en parte indicadas por DeI Carmen (1993), entre las cuales podríamos citar: ausencia de una visión global de lo que se está enseñando (es decir, no se fomenta el aprendizaje de conceptos básicos y generales), falta de progresión en los contenidos (no se suele establecer un grado de diferenciación adecuado en función del nivel eđucativo de que se trate), escasa relación entre los contenidos que se enseñan (la enseñanza de la nutrición se efectúa de forma compartimentada y sesgada, estudiando cada proceso por separado y prestando más atención a los detalles específicos que al establecimiento de relaciones entre ellos) o planificación de la enseñanza sin tener en cuenta los conocimientos previos de los alumnos y alumnas a que se dirige.

\section{REFERENCIAS BIBLIOGRÁFICAS}

ANDERSON, C.W., SHELDON, T.H. y DUBAY, I. (1990) The effects of instruction on college non-major' conceptions of respiration and photosynthesis. Joumal of Research in Science Teaching, 27 (8), pp. 761-776.

ARNAUDIN, M.W. y MINTZES, J.J. (1985). Students alternative conceptions of the human circulatory system: a cross-age study. Sciencie Education, 69(5), pp. 721-733.

AUSUBEL, D.P. (1978). Psicología cognoscitiva: un punto de vista cognoscitivo. México: Trillas.

BANET, E. y NÚÑEZ, F (1989). Ideas de los alumnos sobre la digestiont aspectos fisiológicos. Enseñanza de las Ciencias, $7(1)$, pp. $35-44$.

BANET, E. y NÚÑEZ, F. (1990). Esquemas conceptuales de los alumnos sobre la respiración. Enseñanza de las Ciencias, $8(2)$, pp. $105-110$.

CABALLER, M.J. y GIMÉNEZ, I. (1992). Las ideas de los alumnos y alumnas acerca de la estructura celular de los seres vivos. Enseñanza de las Ciencias, 10 (2), pp. 172-180. c) Aspectos socioculturales, como la influencia del entorno familiar de los alumnos o el papel de los medios de comunicación pueden determinar la elaboración de concepciones alternativas que difieren claramente del conocimiento científico. En este sentido se expresan autores como Solomon (1987), Driver (1989) o LIorens, De Jaime y Llopis (1989), indicando el papel del lenguaje en el aprendizaje de conceptos.

A partir de las argumentaciones realizadas, parece evidente que el estudio de la nutrición precisa de un diseño conceptual y un enfoque didáctico diferentes de los que se vienen realizando tradicionalmente. En esta línea, se ha elaborado una propuesta didáctica que contempla el diseño conceptual de la nutrición humana de manera global e integrada, a la vez que plantea su enseñanza siguiendo una secuencia acorde con el constructivismo orientado a la consecución del cambio conceptual (Driver, 1988; Posner, Strike, Hewson y Gertzog, 1982, entre otros).

Los resultados obtenidos como consecuencia de su puesta en práctica en aulas de $8^{\circ}$ de EGB muestran que una amplia mayoría de las y los estudiantes participantes en nuestra intervención didáctica establecen relaciones adecuadas entre Ios diversos procesos de nutrición, adquiriendo, al mismo tiempo, una visión global e integrada de dicha función. En un próximo trabajo daremos cuenta de las características de la propuesta didáctica y ofreceremos una mayor concreción de estos resultados.
CABALLER, M. y GIMÉNEZ, I. (1993). Las ideas del alumnado sobre el concepto de célula al finalizar la educación general básica. Enseñanza de las Ciencias, 11(1), pp. 63-68

CAREY, S. (1985). Conceptual change in childhood. Cambridge: MIT Press.

DEL CARMEN, L. (1993). Una propuesta práctica para analizar y reelaborar las secuencias de contenidos. Aula, 10, pp. 5-8.

DREYFUS, A. y JUNGWIRTH, E. (1988). The cell concept of 10 th graders: curricular expectations and reality. International Journal of Science Education, 10, pp. 221-230.

DRIVER, R. (1988). Un enfoque constructivista para el desarrollo del currículo de ciencias. Enseñanza de las Ciencias, $6(2)$, pp. 109-120.

DRIVER, R. (1989). Students' conceptions and the learning of science. International Journal of Science Education, I 1 (5), pp. $481-490$.

DRIVER, R. y ERICKSON, G. (1983). Theories-in-action: some theoretical and empirical issues in the study of students? 
conceptual frameworks in science. Sudies in Science Education, 10, pp. 37-60.

DRIVER, R.,GUESNE, E. y TIBERGHIEN, A. (1985). Children's ldeas in Science. Londres: Open University Press.

ENGEL CLOUGH, E. y DRIVER, R. (1986). A study of consistency in the use of students' conceptual frameworks across different task contexts. Science Education, 70 (4), pp. 473-496.

GAGILIARDI, R. (1988). Cómoutilizar la historia de las ciencias en la enseñanza de las ciencias. Enseñanza de las Ciencias, 6(3), pp. 291-296.

GARCIA 7.AFORAS, A.M. (1991). Estudio llevado a cabo sobre representaciones de la respiración celular en los alumnos de bachillerato y COU. Enseñanza de las Ciencias, 9(2), pp. 129-134.

GEIILERT, E. (1962). Chiluren's conceptions of the content and functions of the human body. Genetic Psychology Monographs, 65, pp. 293-405.

GILBERT, J.K. y SWIF', D. (1985). Towards a Lakatosian Analysis of the Piagetian and Aiternative Conceptions Research Programmes. Science Education, 69(5), pp. 681-696.

HASLAM, F. y'TREAGUST, D.F.(1987). Diagnosing secondary students' misconceptions of photosynthesis and respiration in plants using a two-tier multiple chojce instrument. Journal of Biological Education, 21(3), pp. 203-211.

IIMENEZ ALEIXANDRE, M.P. (1990). Los esquemas conceptuales sobre la selección natural: análisis y propuestas pura un cambio conceptual. Tesis doctoral: Universidad Complutense de Madrid

IJIORENS, J.A., DE JAIME, M.C. y LLOPIS, R. (1989). La función del lenguaje en un enfoque constructivista del aprendizaje de las ciencias. Enseñanza de las Ciencias, 7(2), pp. $111-119$.

LUFFIEGO, M., BASTIDA DE LA CALLE, M.F., RAMOS FERNÁNDEZ, F. y SOTO LOPEZ J. (1991). Propuesta teórica y experimental de un modelo sistémico de evolución conceptual. Madrid: Ministerio de Educación y Ciencia.
NAGY, M. H. (1953). Children's conceptions of some bodily functions. Journal of Genetics Psychology, 83, pp. 199-216.

NÚNEZZ, F. (1994). Constructivismo yenseñanza de las siencias. Aplicación al estudio de la nutrición humana en educacion secundaria obligatoria. Tesis doctoral. Universidad de Murcia.

OSBORNE, R. y FREYBERG, P.(1985). Leaming and science: the implications of "Children's science". Nueva Zelanda: Heinemann Educational.

PÉRE7.DF EULATE, M.L. (1992). Utilización de los conceptos previos de los alumnos en la enseñanza-aprendizaje de conocimientos en biología. La nutrición humana: unapropuessta de cambio conceptual. Tesis doctoral. Universidad del País Vasco. Bilbao

POSNER, G.J., STRIKE, K.A., HEWSON, P.W. y GERTZOG, W.A. (1982). Accomodation of a scientific conception: Toward a theory of conceptual change. Sciencie Education, 66, pp. $211-227$

POZO, J.I., GÓMEZ CRESPO, M.A., IIMÓN, M. Y SANZ SERRANO, A. (1991). Procesos cognitives en la comprensión de la ciencia: las ideas de los adolescentes sobre la quimica. Madrid: CIDE/Ministerio de Educación y Ciencia.

SERRANO, T. (1993). El desarrollo conceptual del sistema nervioso en ninos de 5 a 14 años. Modelos mentales. Enseñana de las Ciencias, $11(3)$, pp. 349-351.

SEYMOUR, J. y LONGDEN, B. (1991). Respiration - that's breathing, isn't it? Journal of Biological Education, 25(3), pp. 177-183.

SOLOMON, J. (1987). Social influences on the construction of pupils' understanding of science. Studies in Science Education, 14 , pp. 63-82.

TREAGUST, D.F. (1988). Development and use of diagnostic test to evaluate students' misconceptions in science. International Journal Science Education, 10(2), pp. $159-170$.

YARROCH, W.L. (1991). The implications of content versus item validity on science tests. Journal of Research in Science Teaching, 28(7), pp. 619-630. 


\section{ANEXO I}

Ejemplo de ítems incluidos en el cuestionario.

l. Una vez que la sangre, con todas las sustancias que transporta, llega a los órganos (como los músculos, por ejemplo), ¿qué ocurre? (Escribe V o F según sea verdadero o falso lo que se dice en cada frase.):

La sangre circula en el interior de los vasos sanguíneos
(arterias, capilares) que llegana los órganosias sustancias
nutritivas que leva la sangre no salen de los vasos para
quedarse en los organos.

¿He entendido la pregunta?
口Sí.
$\square$ A MEDIAS.

$\square$ NO.

$\square$ Estoy seguro de mi respuesta.

$\square$ Estoy casi seguro de mi respuesta.

$\square$ Estoy poco seguro de mi respuesta.

$\square$ He contestado al azar. 
2. Al terminar de correr una carrera de 200 metros, respiramos más rápido:

\begin{tabular}{|l|l|} 
iA cuál de estas causas crees que se debe? \\
$\square$ Algunos órganos del cuerpo necesitan más oxigeno. \\
$\square$ No son algunos órganos del cuerpo los que necesitan \\
oxigeno, sino que lo necesitan los pulmones. \\
$\square$ Es la sangre quien necesita oxígeno.
\end{tabular}

¿He entendido la pregunta?

$\square$ sí.

A MEDIAS.

$\square$ NO.

$\square$ Fstoy seguro de mi respuesta.

$\square$ Estoy casi seguro de mi respuesta.

$\square$ Estoy poco seguro de mi respuesta.

$\square$ He contestado al azar. 